\title{
Quasiparticles and anomalous temperature dependence of the low-lying states in the colossal magnetoresistant oxide $\mathrm{La}_{2-2 x} \mathrm{Sr}_{1+2 x} \mathrm{Mn}_{2} \mathrm{O}_{7}(x=0.36)$ from angle-resolved photoemission
}

\author{
S. de Jong, ${ }^{1, *}$ Y. Huang, ${ }^{1}$ I. Santoso,${ }^{1}$ F. Massee, ${ }^{1}$ R. Follath,${ }^{2}$ O. Schwarzkopf,${ }^{2}$ L. Patthey, ${ }^{3}$ M. Shi, ${ }^{3}$ and M. S. Golden ${ }^{1, \dagger}$ \\ ${ }^{1}$ Van der Waals-Zeeman Institute, University of Amsterdam, NL-1018XE Amsterdam, The Netherlands \\ ${ }^{2}$ BESSY GmbH, Albert-Einstein-Strasse 15, 12489 Berlin, Germany \\ ${ }^{3}$ Paul Scherrer Institute, Swiss Light Source, CH-5232 Villigen, Switzerland
}

(Received 10 October 2007; published 18 December 2007)

\begin{abstract}
After years of research into colossal magnetoresistant (CMR) manganites using bulk techniques, there has been a recent upsurge in experiments directly probing the electronic states at or near the surface of the bilayer CMR materials $\mathrm{La}_{2-2 x} \mathrm{Sr}_{1+2 x} \mathrm{Mn}_{2} \mathrm{O}_{7}$ using angle-resolved photoemission or scanning probe microscopy. Here, we report temperature-dependent, angle-resolved photoemission data from single crystals with a doping level of $x=0.36$. The first important result is that there is no sign of a pseudogap in the charge channel of this material for temperatures below the Curie temperature $T_{C}$. The data show unprecedented sharp spectral features, enabling the unambiguous identification of clear, resolution-limited quasiparticle features from the bi-

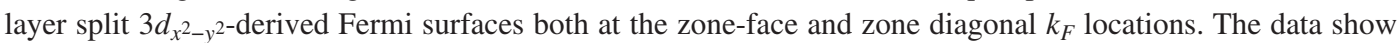
that these low temperature Fermi surfaces describe closed shapes in $k_{\|}$, centered at the $(\pi / a, \pi / a)$ points in the two dimensional Brillouin zone, and are not open and arclike in nature. The second important result concerns the temperature dependence of the electronic states. The spectra display strong incoherent intensity at high binding energies and a very strong temperature dependence, both characteristics reminiscent of polaronic systems. However, the clear and strong quasiparticle peaks at low temperatures are difficult to place within a polaronic scenario. A careful analysis of the temperature-dependent changes in the Fermi surface spectra both at the zone face and zone diagonal regions in $k$ space indicates that the coherent quasiparticle weight disappears for temperatures significantly above $T_{C}$ and that the $k$ dependence of the $T$-induced changes in the spectra invalidates an interpretation of these data in terms of the superposition of a "universal" metallic spectrum and an insulating spectrum whose relative weight changes with temperature. In this sense, our data are not compatible with a phase separation scenario.
\end{abstract}

DOI: 10.1103/PhysRevB.76.235117

PACS number(s): 74.25.Jb, 75.47.Lx, 79.60.-i

The bilayered, strontium doped manganites $\left(\mathrm{La}_{2-2 x} \mathrm{Sr}_{1+2 x} \mathrm{Mn}_{2} \mathrm{O}_{7}\right)$ with $\quad x \approx 0.3-0.4 \quad$ (abbreviated as LSMO) show on cooling an insulator-metal transition associated with the onset of long range ferromagnetic order. This transition occurs at a maximum Curie temperature $T_{C}$, of approximately $130 \mathrm{~K}$ (for $x=0.36$ ), ${ }^{1,2}$ and goes paired with colossal changes in the magnetoresistance (CMR), ${ }^{3}$ which, in turn, have been linked to the large number of spin and orbital degrees of freedom accessible to the near-Fermi-level electronic states. Despite years of research, the microscopic origin of the colossal magnetoresistance effect in these systems is still the subject of much debate.

In general, metallic electrical transport requires the existence of a Fermi surface (FS) upon which quasiparticles (QPs) reside. In this context, LSMO is remarkable, as recent angle resolved photoemission spectroscopy (ARPES) experiments deep in the ferromagnetic, metallic state indicate the existence of two highly differing regimes. Firstly, for $x$ $=0.40,{ }^{4}$ LSMO is reported to possess a discontinuous FS in the form of arcs centered upon the Brillouin zone diagonal (ZD), with a strong pseudogap opening up toward the Brillouin zone face (ZF). In contrast, at an only $2 \%-4 \%$ lower doping level of $x=0.36-0.38,{ }^{5}$ the published spectra exhibit clear quasiparticles at the $\mathrm{ZF}$, leaving open the issue as to the situation at the ZD.

A second, equally important issue is the temperature dependence of the QPs. Seeing that the metallic behavior in LSMO is symbiotic with the ferromagnetic order, one would expect coherent QPs at the FS of LSMO to disappear at $T_{C}$. This appears to be the case at the ZD in crystals with $x$ $=0.40{ }^{4}$ Therefore, a recent report proposing both that ZFQPs exist well above $T_{C}$ for $x=0.36$ (Ref. 6) and that metallic behavior is seen in the ARPES spectra up to a $T^{*}$ of $300 \mathrm{~K}$ has stimulated much discussion.

The layered manganites play a special role within the CMR family, as they enable exploitation of powerful, direct experimental probes of the electronic states in both real [scanning tunneling microscopy (STM)/scanning tunneling spectroscopy] and reciprocal space (ARPES). Therefore, it is of paramount importance that the two central issues sketched above of whether the FS of LSMO is pseudogapped below $T_{C}$ and whether QPs exist above $T_{C}$ are investigated in detail.

This paper presents a thorough ARPES study of these key issues in LSMO single crystals with $x=0.36$. Improved sample quality means we are now able to unambiguously show the existence of QPs at the zone face for both the antibonding and bonding $c$-axis bilayer split $3 d_{x^{2}-y^{2}}$ bands and crucially, also for the ZD direction in the same samples,

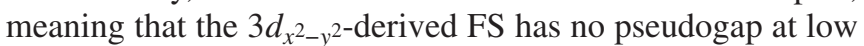
temperatures. Furthermore, a careful analysis of the $k$ dependence of the data recorded at different temperatures provides strong evidence against a scenario of phase separation into microscopic metallic and insulating "patches" above $T_{C}{ }^{6}{ }^{6}$

Experiments used the UE112-PGMa beamline at BESSY coupled to an SES100 analyzer and the SIS beamline at the Swiss Light Source (SLS) equipped with an SES2002 ana- 


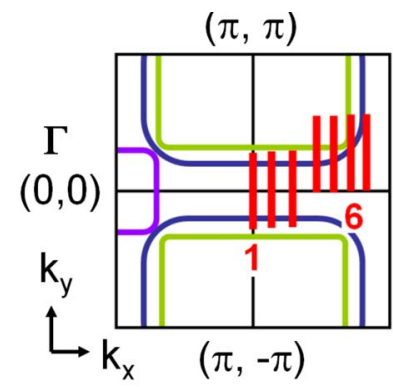

(a)

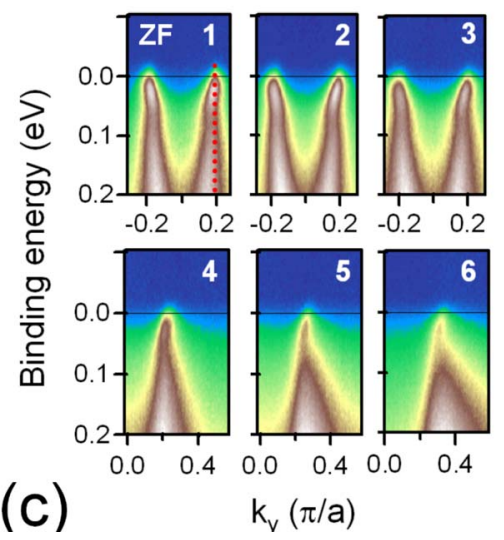

(b)
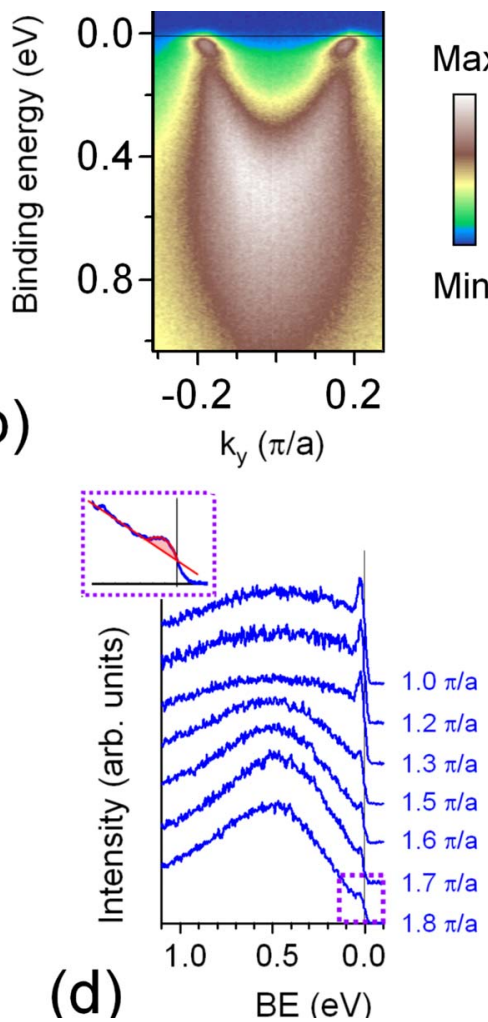

Max

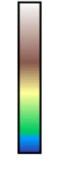

Min

FIG. 1. (Color online) (a) Schematic of the FS

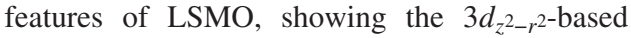
$\Gamma$-centered pocket (purple) and the zone cornercentered FS barrels from the $3 d_{x^{2}-y^{2}}$-based bonding (green) and antibonding (blue) bilayer split bands. The $k$-space cuts taken are marked in red. (b) $(E, k)$ image from LSMO with $x=0.36$ taken at the $(\pi, 0)$ point in $k$ space [cut 1 in panel (a)]. (c) Zoomed images taken at the $k$ slices shown in panel (a). (d) $k_{F}$ EDCs from the images shown in (c). The inset (highlighted in purple) shows a zoom of the EDC for $k_{x}=1.8 \pi / a$. The red shading emphasizes that this EDC also supports a small QP peak on top of a sloping background. All data are recorded with $h \nu=56 \mathrm{eV}$ and $T$ $=30 \mathrm{~K}$.

lyzer and a $50 \mu \mathrm{m}$ sized light spot ( $\mu$-ARPES). The total experimental energy broadening at $25 \mathrm{~K}$ was $30 \mathrm{meV}$ at BESSY and $20 \mathrm{meV}$ at the SLS. The momentum resolution was $0.01 \pi / a$ at the excitation energies used. Single crystals of LSMO were grown in Amsterdam using the optical floating zone technique. The Curie temperature of the samples was determined using superconducting quantum interference device magnetometry to be $131 \mathrm{~K}$. Prior to measurement, the crystals were cleaved at $T<40 \mathrm{~K}$ in a base pressure of 1 $\times 10^{-10}$ mbar. Very sharp tetragonal low energy electron diffraction patterns were obtained from all the measured cleavage surfaces.

A typical $(E, k)$ image, taken at cut 1 of Fig. 1(a), is shown in Fig. 1(b). A "U-shaped" band-in this case the $3 d_{x^{2}-y^{2}}$ antibonding $(\mathrm{AB})$ bilayer-split band-disperses from a band-bottom located at $\approx 450 \mathrm{meV}$ toward the Fermi level, where each branch displays a sharp feature at low energies indicative of the existence of QPs. In panels (c) and (d), the $(E, k)$ images and energy distribution curves (EDCs) from $k_{F}$ locations on the FS are shown. In the former, it is evident that low energy spectral weight survives all the way from the ZF to the ZD region of $k$ space. ${ }^{7}$ In panel (d), the QPs show up as a small, yet clearly discernible peak at $E_{F}$, followed by a hump, at least part of which is due to emission from the deeper lying bonding (B) band (see Fig. 2 and Ref. 8).

If one takes the presence of low energy spectral weight plus a peaked EDC as a working definition of a QP signal, it is evident from Fig. 1 that the $d_{x^{2}-y^{2}}$ bands support QPs all round the FS. Consequently, for $x=0.36$, these FSs are not zone-diagonal "Fermi arcs" and do not support a pseudogap for $T<T_{C}$. This is quite unlike the situation reported for $x$ $=0.40,{ }^{4}$ thus making the pseudogap behavior for this latter doping level not typical for the layered manganites in general.

On a quantitative level, the clear reduction of the QP peak intensity on going from the $\mathrm{ZF}$ to the $\mathrm{ZD}$ is intriguing. ${ }^{9}$

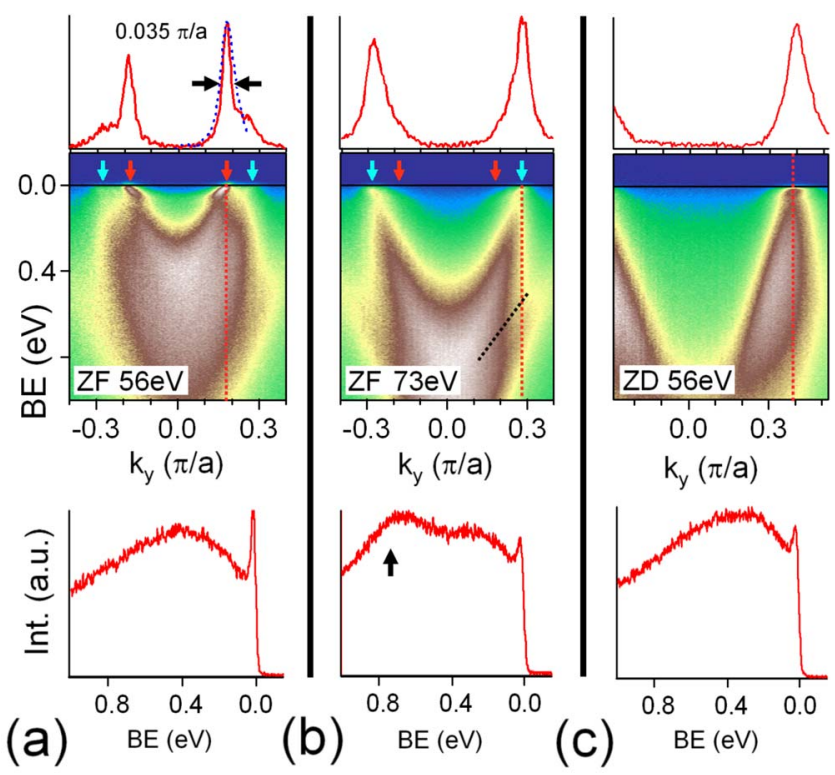

FIG. 2. (Color online) $\mu$-ARPES data for LSMO with $x=0.36$ at $T=30 \mathrm{~K}$ (a) at the zone face with $h \nu=56 \mathrm{eV}$, (b) $h \nu=73 \mathrm{eV}$, and (c) near the zone diagonal, $h \nu=56 \mathrm{eV}$. In each case, the upper panels contain $E_{F}$ MDCs [in panel (a) overlaid with the $E_{F}$-MDC from the data shown in Fig. 1(b) in blue], the central panels the $(E, k)$ images, and lower panels the $k_{F}$ EDCs. The blue and red arrows mark the $k_{F}$ location for the $\mathrm{B}$ and $\mathrm{AB}$ bands, respectively. 
Future studies will be required to explore whether these data can be taken as evidence of coupling to orbiton or phononorbiton degrees of freedom, ${ }^{10}$ which would be expected to be maximal near the zone diagonal, where the $3 d_{z^{2}-r^{2-}}$ and $3 d_{x^{2}-y^{2}}$-derived FSs approach closest to one another. ${ }^{8}$

In the preceding, two criteria were applied to the data to define whether a QP existed or not. Naturally, it is of interest to examine just how well defined these excitations appear to be in both $k$ and $E$. It is known that effective cleavage surface flatness—over the entirety of the illuminated area-can limit the sharpness of ARPES features. The bilayer manganites do not possess a van der Waals bonded cleavage plane as the ARPES and STM "standard" oxide $\mathrm{Bi}_{2} \mathrm{Sr}_{2} \mathrm{CaCu}_{2} \mathrm{O}_{8}$ does, and therefore measurements on LSMO utilizing a highly focused "micro excitation" spot could deliver better defined QP features.

Figure 2 shows $(E, k)$ images recorded using the $50 \mu \mathrm{m}$ light spot at the SLS, together with their corresponding $E_{F}$ momentum distribution curves (MDCs) (upper panels) and $k_{F}$ EDCs (lower panels). It is clear from the $(E, k)$ image in (a) that the qualitative features are very similar to those of Fig. 1(b). The big advantage of the " $\mu$-ARPES" approach can be seen clearly in the top panel of Fig. 2(a). Here, the $E_{F}$ MDCs at $(\pi, 0)$ for the conventional and microspot measurements are overlaid. The full width at half maximum (FWHM) of the leading peak of the former is of the order of $0.06 \pi / a$, a value equal to the state of the art in the literature (Refs. 4 and 5). The small spot data are almost two times narrower, with a FWHM of only $0.035 \pi / a$. The corresponding $k_{F}$ EDC shows a more pronounced peak at $E_{F}$, whose width is essentially resolution limited, as would be expected for a quasiparticle.

Figure 2(b) shows the same $(\pi, 0)$ cut for $h \nu=73 \mathrm{eV}$. This photon energy is reported to strongly favor emission from the B band, ${ }^{5}$ and indeed a further U-like feature is seen, with a band bottom located at $\sim 800 \mathrm{meV}$ and $k_{F}$ wave vectors some $0.1 \pi / a$ greater than those for the $\mathrm{AB}$ band. The $\mathrm{B}$ band $k_{F}$ EDC shows a small, narrow peak at $E_{F}$ signaling the QP, followed by two humplike features at higher energy.

Consideration of both panels (a) and (b) of Fig. 2 reveals a surprising feature of the $\mu$-ARPES measurements on LSMO in the form of an extra, V-shaped, weak feature indicated in the $(E, k)$ image of Fig. 2(b) with a black dotted line. This feature disperses in a parabolic manner around the $(\pi, 0)$ point (as do the $\mathrm{AB}$ and $\mathrm{B}$ bands). From an extrapolation, its $k_{F}$ exceeds $0.5 \pi / a$, a value that is far from matching any FS crossing in band structure calculations. ${ }^{8}$ At present the origin of this feature, which gives rise to the high energy hump in the EDC indicated with an arrow in Fig. 2(b), is unknown. Both the simplicity of the observed tetragonal low energy electron diffraction patterns for the cleaved surfaces and the $k$-space location argue against a simple "diffraction replica" origin.

Figure 2(c) shows data taken in the region of the ZD with $h \nu=56 \mathrm{eV}$. Once more, a sharp QP peak is seen at $E_{F}$, whose intensity, although greater than in the conventional measurement shown in Figs. 1(c) and 1(d), is still small compared to that seen at the ZF for the same photon energy.

Given the identification of well-defined QP features for

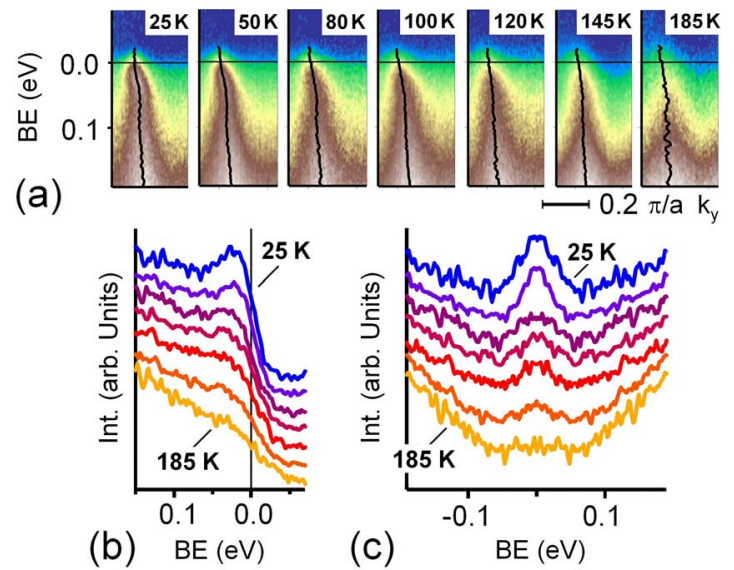

FIG. 3. (Color online) (a) $T$ dependence for LSMO $x=0.36$ at $(\pi, 0)(h \nu=56 \mathrm{eV}$, left hand branch). The MDC peak maxima are overlaid using a black solid line. (b) EDCs at $k_{F}$ for all temperatures, normalized to the intensity at $150 \mathrm{meV} \mathrm{BE}$ and (c) their symmetrized versions (all EDCs are offset vertically).

both $(\pi, 0)$-centered FSs, one can then move on to examine their nesting characteristics. Figure 1(c) shows significant nesting in the $\mathrm{ZF}$ region for the $\mathrm{AB}$ band (the $k_{F}$ vectors remain constant over a fair range in $k_{x}$ ). The nesting vector (expressed in units of $2 \pi / a$ ) is simply the $k_{F}$ value of $q$ $=0.19$. For the $\mathrm{B}$ band at $(\pi, 0)$, the analogous value would be $q=0.28$, making it a much closer match with $q$ vectors at which Jahn-Teller correlations above $T_{C}$ give broad regions of incommensurate $\mathrm{x}$-ray scattering centered at $( \pm \epsilon, 0, \pm 1)$ with $\epsilon=0.3 .^{11}$ This may lie behind the significantly weaker intensity of the QP feature for the B band EDC as compared to the analogous $\mathrm{AB}$ band EDC seen in Fig. 2, although one should bear in mind that the polaron correlations seen in x-ray experiments collapse for $T<T_{C}{ }^{11}$

To summarize this part of the results, the observation of persistent and resolution-limited QP signals at all $k_{F}$ locations probed on the $3 d_{x^{2}-y^{2}}$-derived FSs using $\mu$-ARPES measurements is not only fully consistent with nongapped $(\pi, 0)$-centered FSs in LSMO with $x=0.36$ at low temperature, but also sets the standard for the sharpest photoemission data on the bilayer manganites recorded to date.

We now turn our attention to the issue of the temperature dependence of the Fermi surface electronic states. Figure 3 shows data from the $(\pi, 0)$ point taken at temperatures from 25 to $185 \mathrm{~K}$, the latter well above the $T_{C}$ of $131 \mathrm{~K}$. Panel (a) contains EDMs showing one of the branches of the $\mathrm{AB}$ band. It is clear that-apart from thermal broadening-all the EDMs look comparable. It should be noted that the spectral weight of the QP peak does decrease steadily with temperature, as can be seen from the EDCs shown in Fig. 3(b). We note here that this decrease in intensity with higher temperatures is larger than the change in thermal broadening can account for on going from 30 to $185 \mathrm{~K}$. Nevertheless, even at $185 \mathrm{~K}$, some spectral weight at $E_{F}$ still remains as a step in the EDC. None of the spectra in Fig. 3 show a gap within the experimental resolution and the accuracy of the energy referencing used. This can be seen from the symmetrized $k_{F}$-EDCs displayed in panel (b), although the peak in the 
$185 \mathrm{~K}$ symmetrized spectrum is very small and this EDC might actually be close to being gapped, hinting that a true gap in the charge sector might be opening at even higher temperatures. The fact that spectral intensity at $E_{F}$ near $(\pi, 0)$ exists even $50 \mathrm{~K}$ into the paramagnetic region of the phase diagram ${ }^{6}$ seems at odds with the globally insulating transport characteristic at these elevated temperatures of bilayered LSMO with $x=0.36$. Therefore, drawing together what our $T$-dependent data show us up to this point: (i) The QP peak at lowest energy disappears at or very close to the bulk Curie temperature, yet (ii) at the zone face, there remains (nonpeaked) spectral weight at $E_{F}$ until temperatures of the order of $50 \mathrm{~K}$ above $T_{C}$.

To further investigate this anomalous temperature dependence, spectra were taken with improved statistics and over a wider binding energy range, both the $\mathrm{ZF}$ and $\mathrm{ZD}$ regions of the $(\pi, \pi)$-centered Fermi surfaces at low temperature and just below and above $T_{C}$. These data are shown in Fig. 4. Panel (a) contains the (zoomed) EDMs and (b) the accompanying $k_{F}$ EDCs.

In panels (c) and (d) of Fig. 4, symmetrized versions of the EDCs are depicted, shown in panel (b). Although, as asserted below, the low binding energy coherent spectral weight disappears steadily with increasing temperature at both $k$ points, the symmetrized spectra show that at the ZF and the ZD, again, no gap in the charge sector opens at temperatures up to $140 \mathrm{~K}$.

One striking feature in these $T$-dependent data, both at the $\mathrm{ZF}$ and $\mathrm{ZD}$ regions, is that the aforementioned reduction in spectral weight extends over an energy scale of up to $700-800 \mathrm{meV}$, which is obviously an energy range far in excess of the change in thermal energy. Such large changes have been reported previously for the ZF region in Ref. 6, and their $T$ dependence has been put forward as an argument for microscopic phase separation, whereby local metallic and insulating regions change their relative abundance steadily with temperature. Vital for this model is that the difference spectra between pairs of $k_{F}$ EDCs (recorded at different temperatures) can be collapsed onto a single, universal "metallic EDC." The two difference spectra from our data recorded from the ZF region are shown in Fig. 4(e). At first glance, they do look to be scaled versions of one another, but a closer inspection reveals that the weight of the QP peak in the original EDCs decreases much faster between 95 and $145 \mathrm{~K}$ than it did between 30 and $145 \mathrm{~K}$, meaning that the difference spectra lose their low energy peak as temperature increases. As illustrated in the inset of Fig. 4(e), this decrease in spectral weight of the QP in the difference spectra persists - though less prominently - if the original EDCs taken at 30 and $95 \mathrm{~K}$ are first broadened using an additional Fermi-Dirac distribution (matching the temperature broadening of the spectra recorded at $145 \mathrm{~K}$ ) before the subtraction takes place. This proves that the decrease in QP spectral weight cannot be attributed to temperature broadening alone. If, for the sake of argument, one were to assign the difference spectra in Fig. 4(e) to a metallic phase, as was done in Ref. 6, the data here show clearly that this metallic spectrum loses its coherent QP spectral weight as the temperature climbs above $T_{C}$.

An additional, powerful test of this phase separation sce-
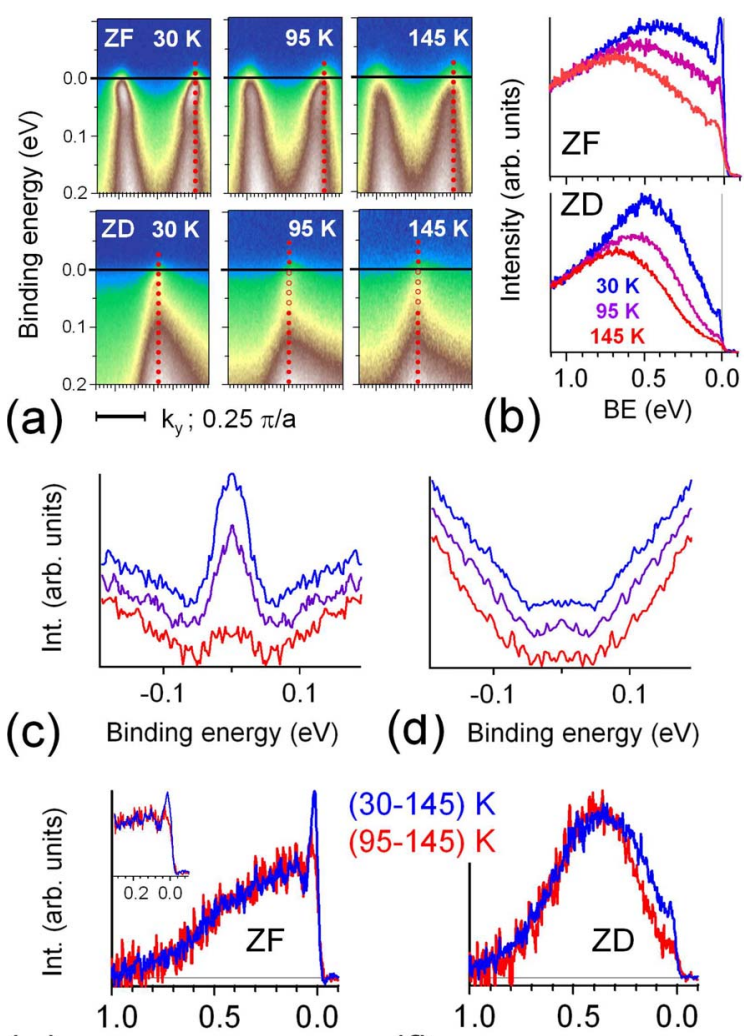

(e) Binding energy (eV)

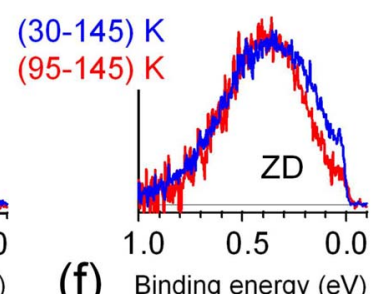

FIG. 4. (Color online) (a) EDMs of LSMO $\left(h \nu=56 \mathrm{eV}, k_{x}\right.$ $=1.0 \pi / a)$ at the $\mathrm{ZF}$ and $\mathrm{ZD}$ point in $k$ space at three different temperatures. (b) EDCs at $k_{F}$ from the EDMs shown in panel (a) (indicated there with red dotted lines). EDCs are normalized to the high $\mathrm{BE}$ intensity, which is equivalent to normalization to the intensity above $E_{F}$. (c) Symmetrized EDCs near $E_{F}$ (scaled at the intensity at $200 \mathrm{meV} \mathrm{BE}$ ) for the three temperatures at the $\mathrm{ZF}$ and (d) at the ZD. (e) The scaled difference between the 30 and $95 \mathrm{~K}$ EDC (blue curve) and the 95 and $145 \mathrm{~K}$ EDC (red curve) for the ZF. The inset shows the same spectra over $300 \mathrm{meV}$ BE for EDCs that are first broadened to match the Fermi-Dirac distribution at $145 \mathrm{~K}$ before subtraction (see text). (f) The scaled difference EDCs of 30-145 K EDC (blue curve) and 95-145 K EDC (red curve) for the ZD.

nario is given by the $k$ dependence of the spectral changes with temperature. If temperature essentially only alters the relative percentage of the metallic and insulating patches, then various difference spectra at a FS location other than the ZF should also collapse onto a universal metallic EDC. From Figs. 1 and 2, it is clear that LSMO with $x=0.36$ also supports QPs in the zone-diagonal region, thus offering the chance of an independent test of the phase separation scenario.

The ZD difference spectra shown in Fig. 4(f) clearly differ significantly up to binding energies of $300 \mathrm{meV}$; thus, one cannot speak of a universal metallic EDC growing monotonically with decreasing temperature for the $\mathrm{ZD}$ region of $k$ space. This, in turn, provides a strong argument against the phase separation model presented in Ref. 6 as being a complete description of the $T$ dependence of these $k$-dependent data, as this scenario would demand that the change in spec- 
tral weight happens in a similar fashion at both high symmetry points of the Brillouin zone. At this stage, it is interesting to recall that a recent $T$-dependent STM study of LSMO with $x=0.30$ (Ref. 12) found no signs of a bimodal gap distribution in tunneling data across more than 2000 different surface locations, which-taken at face value-also argues against phase separation.

Thus, the challenge faced is to reconcile the following four main characteristics of these highly resolved LSMO data for $x=0.36$ :

(i) existence of clear and sharp QP peaks at all $3 d_{x^{2}-y^{2} \text {-derived } k_{F}}$ locations probed at low $T$;

(ii) strong high binding energy (BE) spectral weight at all temperatures;

(iii) $T$-dependent changes in the spectra on a scale of up to $800 \mathrm{meV} \mathrm{BE}$;

(iv) clear differences in the temperature induced effects between the $\mathrm{ZF}$ and $\mathrm{ZD}$ regions of $k$ space.

Phase separation as described above would have offered a route to reconcile points (i)-(iii), but cannot account for point (iv). On the other hand, behavior such as described in points (ii)-(iv) has been reported for photoemission from polaronic systems, ${ }^{13,14}$ in which much of the higher binding energy intensity is due to multiphonon satellites, and a vanishingly weak QP (the "zero phonon line") is left at $E_{F}$. The kind of electron-phonon coupling strengths required to generate all the observed high $\mathrm{BE}$ incoherent weight ${ }^{15}$ seen in these data from LSMO would lead to the complete suppression of the QP feature at $E_{F}$, something that evidently does not occur here for $T \ll T_{C}$ [point (i), above].

From the above, it is apparent that some pieces of the puzzle that are required for a complete and consistent description of the ARPES data from these systems (and their temperature dependence) must still be missing. Despite this pointer toward future work in this area, the data do result in a number of unambiguous and important conclusions. First, the $(\pi, \pi)$-centered Fermi surfaces of the layered CMR manganite $\mathrm{La}_{1.28} \mathrm{Sr}_{1.72} \mathrm{Mn}_{2} \mathrm{O}_{7}$ support quasiparticles, both at the diagonal and the face of the 2D Brillouin zone at low temperatures. This system, therefore, has no pseudogap in the charge sector, thereby excluding the use of the epithet "nodal metal" for this class of materials in general. Second, we find that the temperature-dependent behavior of both the quasiparticles and of higher lying spectral weight is different at the Brillouin zone face and diagonal. This argues against a model describing the metal-insulator transition as stemming from a percolative growth of metallic clusters in an insulating matrix.

We thank the IFW Dresden group for lending us their spectrometer and W. Koops and T. J. Gortenmulder for expert technical support. This work was funded by the FOM (ILP and SICM) and the EU (via I3 Contract No. RII3-CT2004-506008 at both BESSY and SLS). *sdejong@science.uva.nl

†mgolden@science.uva.nl

${ }^{1}$ C. D. Ling, J. E. Millburn, J. F. Mitchell, D. N. Argyriou, J. Linton, and H. N. Bordallo, Phys. Rev. B 62, 15096 (2000).

${ }^{2}$ M. Kubota, H. Fujiokab, K. Ohoyamac, K. Hirotab, Y. Moritomod, H. Yoshizawa, and Y. Endohb, J. Phys. Chem. Solids 60, 1161 (1999).

${ }^{3}$ Y. Moritomo, A. Asamitsu, H. Kuwahara, and Y. Tokura, Nature (London) 380, 141 (1996).

${ }^{4}$ N. Mannella, W. L. Yang, X. J. Zhou, H. Zheng, J. F. Mitchell, J. Zaanen, T. P. Devereaux, N. Nagaosa, Z. Hussain, and Z.-X. Shen, Nature (London) 438, 474 (2005).

${ }^{5}$ Z. Sun, Y.-D. Chuang, A. V. Fedorov, J. F. Douglas, D. Reznik, F. Weber, N. Aliouane, D. N. Argyriou, H. Zheng, J. F. Mitchell, T. Kimura, Y. Tokura, A. Revcolevschi, and D. S. Dessau, Phys. Rev. Lett. 97, 056401 (2006).

${ }^{6}$ Z. Sun, J. F. Douglas, A. V. Fedorov, Y.-D. Chuang, H. Zheng, J. F. Mitchell, and D. S. Dessau, Nat. Phys. 3, 248 (2007).

${ }^{7}$ For the $\mathrm{ZD}$ direction, the bonding and antibonding bilayer split FSs almost touch. Exactly which of these FSs dominates the observed intensity is a moot point.

${ }^{8}$ X. Y. Huang, O. N. Mryasov, D. L. Novikov, and A. J. Freeman, Phys. Rev. B 62, 13318 (2000).
${ }^{9}$ We note here that a reduction of intensity at the ZD in the ARPES experiment could also be (at least partially) the result of (i) matrix element effects, (ii) the fact that the $k$-space cut at the ZD is not perpendicular to the FS, and (iii) the increasing Fermi velocity toward the $\mathrm{ZD}$ region of the Brillouin zone.

${ }^{10}$ J. van den Brink, Phys. Rev. Lett. 87, 217202 (2001).

${ }^{11}$ B. J. Campbell, R. Osborn, D. N. Argyriou, L. Vasiliu-Doloc, J. F. Mitchell, S. K. Sinha, U. Ruett, C. D. Ling, Z. Islam, and J. W. Lynn, Phys. Rev. B 65, 014427 (2001).

${ }^{12}$ H. M. Rønnow, Ch. Renner, G. Aeppli, T. Kimura, and Y. Tokura, Nature (London) 440, 1025 (2006).

${ }^{13}$ C. Kim, F. Ronning, A. Damascelli, D. L. Feng, Z.-X. Shen, B. O. Wells, Y. J. Kim, R. J. Birgeneau, M. A. Kastner, L. L. Miller, H. Eisaki, and S. Uchida, Phys. Rev. B 65, 174516 (2002).

${ }^{14}$ K. M. Shen, F. Ronning, D. H. Lu, W. S. Lee, N. J. C. Ingle, W. Meevasana, F. Baumberger, A. Damascelli, N. P. Armitage, L. L. Miller, Y. Kohsaka, M. Azuma, M. Takano, H. Takagi, and Z.-X. Shen, Phys. Rev. Lett. 93, 267002 (2004).

${ }^{15} \mathrm{We}$ note that the $k_{F}$ EDCs as presented in this paper also contain spectral weight due to bands located at higher BE, for example, the $\mathrm{B}$ band and the third, at present unidentified feature mentioned earlier. 